\title{
Study on the isolated test technologies
}

\author{
Jiang Yang ${ }^{\text {a }}$ \\ Institute of Electronic Engineering, China Academy of Engineering Physics, mianyang, 621900, China
}

\begin{abstract}
This paper discusses the means of electrical isolation especially the characteristics of photoelectric isolation, magnetoelectric isolation and floating ground in common use. The two typical applications are presented based on applied requirement. One is analog testing module which consists of V/F conversion circuit and photoelectric isolation circuit. First analog signals are converted frequency signals and then frequency signals are transferred by photoelectric coupler. It is with low linearity error, high test precision and high isolated voltage. Another is digital signals test module based on isolated transformer. It uses the device which is quadchannel digital isolator with an intergraded isolated dc-to-dc converter. The device provides power supply to tested circuit. The module is small volume and high isolation voltage. Both applications are with practicability by validated with applied.
\end{abstract}

Keywords: Isolated test; photoelectric isolation; magnetoelectric isolation.

\section{Introduction}

In order to avoiding mutually interference and ensuring security and electromagnetic compatibility of two systems, it is necessary that two relatively self-governed systems transmit signals isolated. These signals are analog signals, digital signals and bus data. The means of electric isolation are mainly photoelectric isolated technology[1], magnetoelectric isolated technology[2], electromechanical isolated technology, acoustoelectric isolated technology and floating ground technology. Photoelectric isolation uses photoelectric coupler and magnetoelectric isolation uses isolated transformer.

This paper discusses the means of photoelectric isolated technology, magnetoelectric isolated technology and floating ground in common use. The two typical applications are presented based on application requirements. They are with practicability by validated with applied.

\section{The approach of isolated test}

\subsection{Photoelectric isolated technologies}

\subsubsection{Photoelectric coupler}

The implement of photoelectric isolation come by photoelectric coupler that transmits light by light emitting diode (LED) and receives light by photo semiconductor (photoresistor, photodiode,

\footnotetext{
${ }^{\mathrm{a}}$ Corresponding author : yangj0830@sohu.com
}

(C) 2016. The authors - Published by Atlantis Press 
phototransistor). Because LED and photosemiconductor are mutually insulated the isolation of two systems is realized.

\subsubsection{The characteristics of photoelectric isolation circuit}

The photoelectric isolation circuit characterizes transmitting signal by light other than current and voltage. According to the characteristic it is merely considered that input and output impedance of photoelectric isolation circuit rather than impedance matching between two circuits when photoelectric isolation circuit is designed and used.

When the photoelectric coupler is used it is unnecessary to consider the effect of two circuit connecting and more the effect of direct current potential. For phototransistor the front input circuit potential provides for the potential of light emitting diode, which produces direct current that offers base potential of phototransistor through photoelectric conversion.

The connecting form between two circuits depends on the used photoelectric coupler and implemented function. It is pay attention the follow basic request. When analog signal is transferred the index of linearity and frequency is need to satisfied the requirement. If used LED is incapable of content condition of linearity, the nonlinear distortion occurs for transferred signal. Moreover if used coupler is incapable of content condition of bandwidth, the nonlinear distortion occurs for transferred signal. In order to eliminate diode nonlinear the base voltage is commonly offered for diode to run in linear region, which called static state run voltage.

\subsubsection{Photoelectric coupling circuit applications}

Because input resistance of photoelectric coupler is less than impedance of disturbance source the disturbing voltage in input of photoelectric coupler is lower, and so is disturbing current in input of photoelectric coupler. It is unable to make LED producing light. Photoelectric coupler will not be affected by outside light due to its sealed shell. The insulating resistance of photoelectric coupler is higher $(1012 \Omega)$ and the insulating capacitance of photoelectric coupler is lesser (two or three $\mathrm{pF}$ ), therefore it can prevent electromagnetic disturbance by electrocircuit coupling. The insulating resistance of photoelectric coupler is diminished along with increasing of working frequency, so is the effect of anti-interference.

\subsection{Magnetoelectric Isolated technologies}

\subsubsection{Basic principle of magnetoelectric isolation by transformer}

The transformer is mainly composed of two or several coil windings in common iron-core. When alternating voltage apply to one of coil windings, the alternating voltage is induced in other coil windings by electromagnetic induction. Thus among several coil windings interact each other by alternating magneticfield and they are isolated in circuit. The dielectric strength of isolation is dependent on insulating strength among several coil windings and grand.

\subsubsection{Actual transformer}

When operating frequency is high the skin effect would lead to increase in winding resistance and in heating loss. Leakage flux tends to disturb device and wire near by actual transformer it ought to select transformer about low leakage flux and strengthen in magnetic field shield.

The high frequency disturbance getting in electrical source transformer primary port couples to secondary port by parasitic capacitance between primary and secondary ports. When static shield is added between electrical source transformer primary port and secondary port the new parasitic capacitance is formed between static shield and windings. The high frequency disturbance will be 
returned to ground via the new parasitic capacitance when static shield grounding. This will carry out anti-interference.

Insulating strength among winding and ground is dependant on isolated voltage resistance which involves operating voltage, variation in voltage, transient state overvoltage, and surplus voltage.

Operating frequency not only affects iron-core loss of transformer but also is mutually related to impedance of transformer. For instance, the impedance of inductance $\mathrm{L}$ is direct ratio to operating frequency and in turn the impedance of capacitance $C$ is inverse ratio to operating frequency. The magnetoelectric isolation by transformer goes together with shielding and grounding technology when parasitic capacitance of transformer winding is bigger.

\subsection{Floating ground technology}

\subsubsection{Floating ground}

Floating ground means that the circuit ground doesn't connect to earth ground. Its advantage is that the circuit isn't affected by electric performance of earth ground and its disadvantage is that the circuit is likely to be affected by parasitic capacitance and the circuit's electric potential is likely to change, which will induce interference for analog circuit.

Floating ground make the isolated resistance bigger which can prevent the electromagnetic interference of common-ground resistance.

\subsubsection{Floating ground technology application}

a) Dividing alternating current ground from direct current ground

The alternating current source ground generally connects to earth ground. There is grounding resistance and there is current on it. This led to that zero line electric potential of alternating current source is not zero potential. In addition there is a lot of interference on zero line of alternating current source. If alternating current ground does not divide from direct current ground the interference could affect direct current source and direct current circuit running normally. Therefore dividing alternating current ground from direct current ground could isolate interference from alternating current source earth line.

b) Amplifier floating ground technology

For amplifier especially small signal input and high gain amplifier, any small interfere signal may lead to running abnormally. Thus adopting amplifier floating ground technology could prevent interference signal entering and improve electromagnetic compatibility of amplifier.

\section{The applications of isolated test}

\subsection{Analog signal test based on VIF conversion and photoelectric isolation}

There are isolated amplifier coupling test mode, linear photoelectric coupler test mode, V/F conversion and photoelectric coupling test mode mainly about analog signal test. The isolated amplifier coupling test mode is provided with wider dynamic range, better linearity, but it need independent power supply and isolated transformer and its circuit volume is bigger. Linear photoelectric coupler test mode is endowed with lesser circuit, but it needs superior symmetry and consistency and it is difficulty to choose device and its dynamic range is less. The V/F conversion and photoelectric coupling test mode is composed of $\mathrm{V} / \mathrm{F}$ conversion circuit and photoelectric coupling circuit. Its circuit is simple and with higher linearity.

According to application demand the paper elects V/F conversion and photoelectric coupling test mode. In circuit $\mathrm{V} / \mathrm{F}$ conversion module convert tested analog signal into frequency signal and output square wave. The V/F conversion module is supplied power by tested signal. Through photoelectric 
coupling the single chip realize voltage test by testing frequency. In test the arithmetic mean filtering is adopted to eliminate stochastic disturbance. The principle chart is as figure 1.

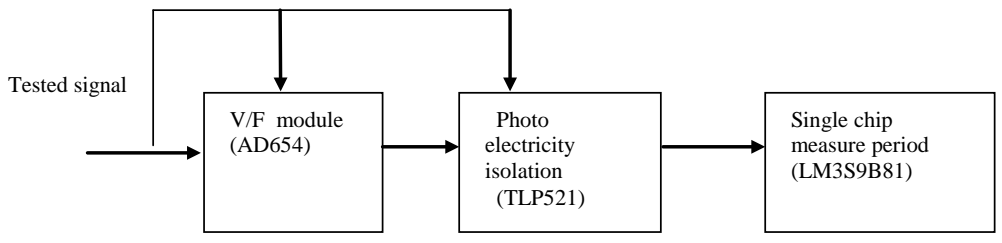

Figure 1. Implementation of isolation measurement

The V/F conversion module uses low cost AD654[4]. The AD654 is a monolithic V/F converter consisting of an input amplifier, a precision oscillator system, and a high current output stage.

The AD654 is a complete V/F converter requiring an RC timing network to set the desired fullscale frequency and a selectable pull-up resistor for the open-collector output stage. Any full scale input voltage range from $100 \mathrm{mV}$ to 10 volts (or greater, depending on +VS) can be accommodated by proper selection of the timing resistor. The full scale frequency is then set by the timing capacitor from the relationship, $\mathrm{f}=\mathrm{V} / 10 \mathrm{RC}$. Power supply requirements are minimal; only $2.0 \mathrm{~mA}$ of quiescent current is drawn from the single positive supply from 4.5 volts to 36 volts. In this mode, positive inputs can vary from 0 volts (ground) to (+VS -4) volts. Negative inputs can easily be connected for below ground operation. The versatile open-collector output stage can sink more than $10 \mathrm{~mA}$ with a saturation voltage less than 0.4 volts. The Logic Common terminal can be connected to any level between ground (or -VS) and 4 volts below +VS. An operational amplifier serves as the input stage; its purpose is to convert and scale the input voltage signal to a drive current in the NPN follower. Optimum performance is achieved when, at the full-scale input voltage, a $1 \mathrm{~mA}$ drive current is delivered to the current-to-frequency converter (an astable multivibrator). The drive current provides both the bias levels and the charging current to the externally connected timing capacitor. This "adaptive" bias scheme allows the oscillator to provide low nonlinearity over the entire current input range of $100 \mathrm{nA}$ to $2 \mathrm{~mA}$.

The photoelectric coupling circuit uses TLP521. The TLP521 series of optically coupled isolators consist of infrared light emitting diodes and NPN silicon photo transistors.

The implementation circuit is as figure 2 .

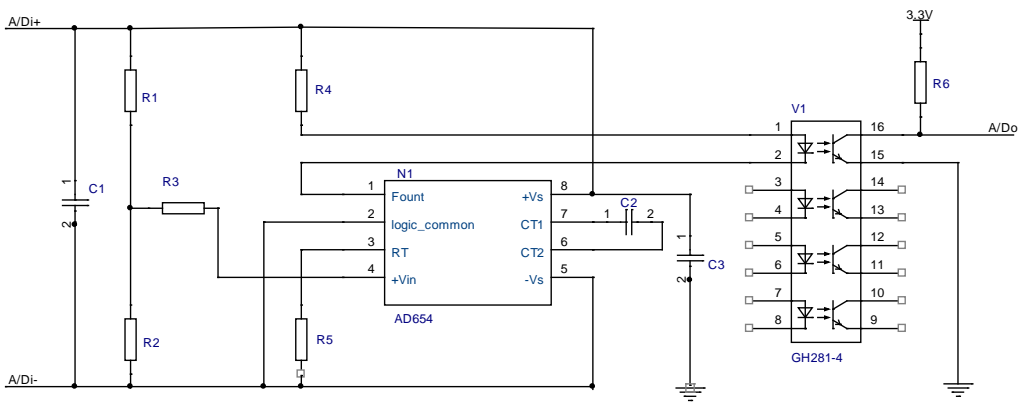

Figure 2. Implementation circuit of isolation test

The tested analog signal (A/D) is divided into two fractions. One connects to +Vs as $\mathrm{V} / \mathrm{F}$ conversion module power supply which can cut down isolated power supply. Another enters into V/F converter input port +Vin through voltage divider R1, R2 and match resistor R3. The voltage divider $\mathrm{R} 1, \mathrm{R} 2$, match resistor R3 and current limiting resistor are selected according to the rule that Optimum performance is achieved when, at the full-scale input voltage, a $1 \mathrm{~mA}$ drive current is delivered to the 
current-to-frequency converter. The time base capacitor C2 is selected according to the rule that 10 $\mathrm{kHz}$ full-scale frequency is achieved when, at the full-scale input voltage, which is fitted the transfer frequency request of photoelectric coupler V1. The output of AD654 is isolated by photoelectric coupler V1, and then enter IO port of single chip microcontroller that measure frequency by the aid of software. In order to improving anti-interference, the common-mode anti-interference capacitor C1 and the differential-common anti-interference capacitor $\mathrm{C} 3$ are placed in the input port of $\mathrm{AD}$ convertor.

\subsection{The on-off state test based on isolated transformer}

In some occasion the on-off state is request to test. For example the normally open contact and normally close contact of switch, the normally open contact and normally close contact of relay. These switches are passive and need test power supply. In addition the test for on-off state is in request to electric isolation between tested circuit and testing circuit for the sake of preventing mutually influence and interference.

According to above application characteristics the digital isolator ADuM5404 is selected[5]. The ADuM5404 device is quad-channel digital isolators with isoPower ${ }^{\circledR}$, an integrated, isolated dc-to-dc converter. The ADuM5404 isolator provides four independent isolation channels in a variety of channel configurations and data rates.

The on-off state test implementation principle chart is as figure 3. The isolator ADuM5404 provides test power supply for tested circuit by isolated dc-to-dc converter. The tested contact state is converted into digital signal and delivers to IO port of single chip microcontroller through isolation channel. IO port of single chip microcontroller implements signal frequency test.

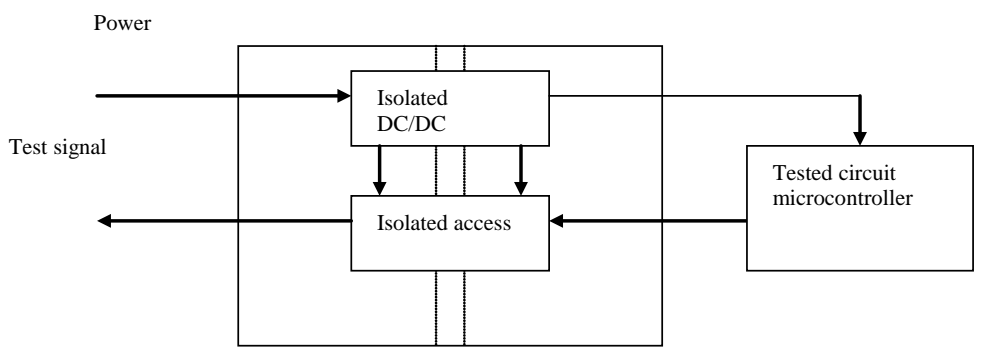

Figure 3. The on-off state test implementation principle chart

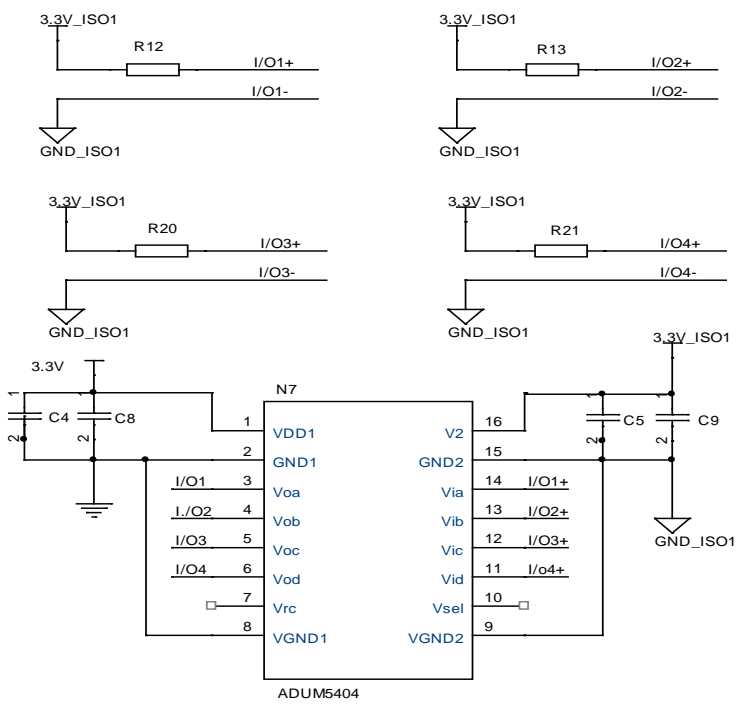

Figure 4. The on-off state test implementation circuits chart 
The on-off state test implementation circuit chart is as figure 4. The 3.3V connects into ADuM5404 power input port VDD1 through filtering capacitors C4, C8. It exports in output port V2(3.3_ISO1)through isolation. In output port filtering capacitors C5, C9 are placed. The power supply 3.3_ISO1 connects to tested passive switch ports(IOx+,IOx-)through current limiting resistors (R12,R13,R20,R21), which provides power supply for tested contact. In the meanwhile tested contacts one port connects ADuM5404 imports (Via,Vib,Vic,Vid), another port connects isolated power supply ground. By coupling the tested contacts state is exported in isolator output ports (IO1,IO2,IO3,IO4). The output signal of isolator enters to IO ports of micro chip controller to test.

\section{Summary}

In order to verify suggested applications environment adaptability, some experiments have made. Include low temperature operation test $\left(0^{\circ} \mathrm{C}\right)$, high temperature operation test $\left(40^{\circ} \mathrm{C}\right)$, damp and hot test, simulation transport test. The modules operate well. The performance index is as table 1 .

Table 1. The performance index

\begin{tabular}{ll}
\hline Index & Value \\
\hline Analog portion isolation voltage & $5.3 \mathrm{KV}$ \\
Digit portion insulation voltage & $2.5 \mathrm{KV}$ \\
Analog portion delay & $11.02 \mathrm{uS}$ \\
Digit portion delay & $140 \mathrm{nS}$ \\
& \\
\hline
\end{tabular}

Above applications can satisfy requirement of application by applied validation. The performance achieves the request of design. It is with practicability. The discussion in paper may be reference for mechatronics.

\section{References}

1. Zhong Tong-min, Brief exploration on the isolation technology of electrical and electronic circuit, technological development of enterprise. Vol.30 No.23 Dec.2011. 35-36.(In Chinese)

2. Guo Yun-song, ZHANG Xiao-guang, ZGANG Ying-yuan, Isolation Technologies in Electromagnetic Compatibility, power supply technologies and applications. Vol.5No.1.2 January 2002. 13-17. (In Chinese)

3. TEXAS INSTRUMENTS, Stellaris ${ }^{\circledR}$ LM3S9B96 Microcontroller DATA SHEET Copyright. 2007-2009 Texas Instruments Incorporated.

4. Analog Devices, Low Cost Monolithic Voltage-to-Frequency Converter AD654, http://www.analog.com

5. Analog Devices, Quad-Channel Isolators with Integrated DC-to-DC Converter ADuM5401/ADuM5402/ADuM5403/ADuM5404, http://www.analog.com 